\title{
Bayesian parameter estimation in the second LISA Pathfinder Mock Data Challenge
}

\author{
M Nofrarias, C Röver, M Hewitson, A Monsky, G Heinzel, and K Danzmann \\ Max-Planck-Institut für Gravitationsphysik (Albert-Einstein-Institut) \\ and Leibniz Universität Hannover, 30167 Hannover, Germany \\ L Ferraioli, M Hueller, and S Vitale \\ Dipartimento di Fisica, Università di Trento, and I.N.F.N., Gruppo di Trento, 38050 Povo, Italy
}

(Dated: April 22, 2022)

\begin{abstract}
A main scientific output of the LISA Pathfinder mission is to provide a noise model that can be extended to the future gravitational wave observatory, LISA. The success of the mission depends thus upon a deep understanding of the instrument, especially the ability to correctly determine the parameters of the underlying noise model. In this work we estimate the parameters of a simplified model of the LISA Technology Package (LTP) instrument. We describe the LTP by means of a closed-loop model that is used to generate the data, both injected signals and noise. Then, parameters are estimated using a Bayesian framework and it is shown that this method reaches the optimal attainable error, the Cramér-Rao bound. We also address an important issue for the mission: how to efficiently combine the results of different experiments to obtain a unique set of parameters describing the instrument.
\end{abstract}

\section{INTRODUCTION}

LISA Pathfinder 1 is an ESA mission, with some NASA contributions, that aims at testing key technologies for the future space gravitational wave observatory, LISA 2]. The main aim is to demonstrate the ability to put a test mass in to free-fall at a level of $3 \times 10^{-14} \mathrm{~ms}^{-2} / \sqrt{\mathrm{Hz}}$ at $1 \mathrm{mHz}$. The LISA Technology Package (LTP) is the main instrument on-board LISA Pathfinder. It comprises two test masses enclosed in inertial sensors which are in turn housed inside individual vacuum tanks, composing the so called Gravitational Reference Sensor 3. The two tanks are then mounted to a support structure which also holds an optical bench between the tanks. The optical bench and the associated interferometry are part of the Optical Metrology System [4. In order to reach the goal stated above, the full LTP must be characterized and optimized. This will involve developing a full parametric noise model of the instrument, which will be improved over the course of the mission.

The LISA Pathfinder mission comprises a series of experiments. Many of the experiments aim to reduce the noise in the system so as to produce the quietest residual acceleration measurement possible. Other experiments will aim to characterize the instrument. This typically involves determining the various parameters that go into the physical model of the instrument. Clearly, a good model is needed to be able to target and reduce particular noise sources, whereas reducing the various noise sources leads to a more sensitive instrument. Various experiments will be repeated under different conditions, and as the noise is reduced, we would expect that the determination of the physical parameters will become more and more accurate. One essential aspect of this multipleexperiment mission is the ability to include the results from analyzing the previous experiments in further experiments, and in particular, it will be necessary to com- bine the various experiments to gain the best knowledge about the particular physical parameters. The analysis procedures and software need therefore to remain flexible in order to react to the results of the experiments as they are performed. This paper presents a Bayesian analysis for determining particular physical parameters of the system. Using a Bayesian framework leads to a natural way of combining a series of experiments. The result of one analysis becomes prior information in subsequent analyses. The analysis is presented for a reduced set of physical parameters in the context of the Mock Data Challenges (MDC) 5] that are being carried out during the development of the data analysis procedures for the mission. In MDC1 [6] the focus was on developing a simple model of the system, together with establishing routines for calibrating the measured test mass displacements back to equivalent residual external test mass accelerations. In MDC2, the focus shifts to parameter estimation. The analysis and procedures presented in this paper represent one of the methods being developed for the mission.

\section{THE SECOND LTP MOCK DATA CHALLENGE}

The aim of the second MDC was to develop and test reliable methods to accurately estimate the parameters of the LTP noise model during flight operations. In order to focus on methods and not on model complexity, it was decided to keep a very similar model as the one analyzed during the first MDC. The basic difference regarding the previous challenge is that now 5 parameters are considered as degrees-of-freedom of the system, which need to be determined by stimulating the system using injected signals. It is worth recalling that the first MDC did not include any signal injection in the data, since it was designed as a test of the calibration of displacement noise to acceleration noise, and therefore only a noise measure- 

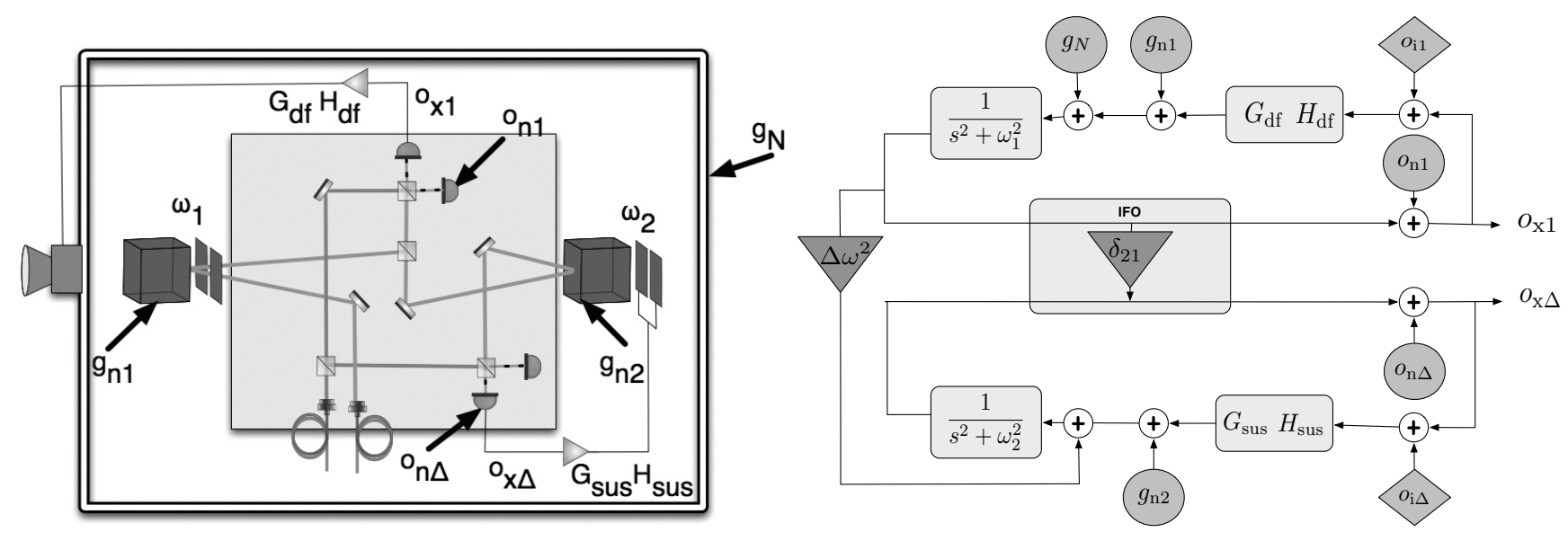

FIG. 1. The LTP MDC2 model. Left: Simplified scheme of the LTP instrument. Only two out of the four heterodyne interferometers are represented here: the one measuring spacecraft to first test mass distance, $o_{x 1}$, and the one measuring test mass to test mass distance, $o_{\Delta x}$. See text for a description of terms appearing in the picture. Right: The previous is described as a control loop: the boxes describe the interferometer (IFO), controllers and dynamics of the test masses. The circles represent noise contributions, diamonds are signal injection points and the triangles denote cross-couplings between the first $\left(o_{x 1}\right)$ and second channel $\left(o_{\Delta x}\right)$.

ment (signal free) was simulated. The current challenge is therefore a natural extension to the first one.

It is important to notice that, due to the nature of the LISA Pathfinder mission, our description of the system necessarily needs to deal with the closed-loop dynamics of the spacecraft and test masses together.

The description that we show in Section IIA is therefore a closed-loop system where we take into account the feedback between different components and show where parameters and noise contributions enter in the nonlinear model that is described in terms of transfer functions in the frequency domain. We want to recall that this approach differs from the one used to model LISA to the date. The data generators that are providing data in the LISA Mock Data Challenges [7-9] are focused on the geometry of the spacecraft configuration, since the main concern is, in that case, the suppression of frequency noise due to the unequal arms. But, on the other hand, they consider additive noise sources inside each spacecraft. A second important remark is that LISA generators model noise sources as white gaussian contributions. This is clearly unrealistic and could be particularly misleading in the relevant region around $1 \mathrm{mHz}$, since each of the noise sources will contribute with a $f^{-p}(p \simeq 1)$ power spectrum that will set the low frequency performance of LISA. LTP is designed to study that region and therefore our model needs to describe these low frequency contributions in more detail. The noise models and the parameters used are described in Section IIB. The description provided in this paper will complement the one already existing within the LISA community and will facilitate the interaction between both communities to a common goal, which is a realistic understanding of the LISA instrument.

In terms of implementation, it is worth mentioning that the current challenge is completely implemented as LTPDA tools [5], which means that any user of this tool has the means available to produce LTP-like data (as described in the following section) by executing a relatively simple MATLAB [10] script.

\section{A. Dynamical model}

When compared with other space missions, the LTP is a very flexible instrument in terms of the possible operational scenarios. It can be configured to use different combinations of the available sensors onboard, either optical or capacitive, with the aim of performing different geodesic measurements, or even to work as an accelerometer. The aim of the second MDC was not to cover all of these possibilities but to analyze the instrument behavior for a fixed operating mode: the main science mode — described as M3 mode in [11. Moreover, this control scheme is reduced in this analysis to the one-dimensional case in order to simplify the model and focus on the analysis. In this simplified model, the $x$ position of both test masses is controlled by means of the optical readouts. A first interferometer measures the relative distance between test mass 1 and the spacecraft, $\mathrm{x}_{1}$. This is a relatively noisy measurement since the noise of the spacecraft's micro-Newton thrusters appears directly in the measurement. A second interferometer measures the relative distance between both free falling test masses. This channel, that we call $\mathrm{x}_{\Delta}$ in the rest of this paper, will be the one giving an unprecedently quiet measurement of the differential acceleration (or displacement) between two test masses, since the contribution of the thruster noise effectively cancels out 12 .

The model of the LTP dynamics control loop is shown 
in Figure 1. The right panel of this figure shows two control loops for the two measurement channels that we just described: $\mathrm{x}_{1}$ and $\mathrm{x}_{\Delta}$. This schematic representation of the closed loop system can be analytically expressed in terms of the following set of equations [13]

$$
\begin{aligned}
\mathbf{D} \cdot \vec{q} & =\vec{g}, \\
\vec{g} & =-\mathbf{C} \cdot\left(\vec{o}+\overrightarrow{o_{i}}\right)-\overrightarrow{g_{n}}, \\
\vec{o} & =\mathbf{S} \cdot \vec{q}+\overrightarrow{o_{n}},
\end{aligned}
$$

where $\mathbf{D}$ is the dynamical matrix, $\mathbf{C}$ is the controller, and $\mathbf{S}$ stands for the sensing matrix (the interferometer in our case), i.e., the matrix translating the position of a test mass, $\vec{q}$, into the interferometer readout, $\vec{o}$. Subindex $n$ stands for noise quantities, either sensing noise $\left(\vec{o}_{n}\right)$ or force noise $\left(\vec{g}_{n}\right)$ and subindex $i$ stands for the injected signals $\left(\vec{o}_{i}\right)$. All of these are 2 -dimensional vectors with components referring to the $\mathrm{x}_{1}$ and $\mathrm{x}_{\Delta}$ channels respectively,

$$
\begin{array}{r}
\vec{q}=\left(\begin{array}{c}
x_{1} \\
x_{\Delta}
\end{array}\right), \vec{o}=\left(\begin{array}{c}
o_{x 1} \\
o_{x \Delta}
\end{array}\right), \\
\vec{o}_{i}=\left(\begin{array}{c}
o_{i 1} \\
o_{i \Delta}
\end{array}\right), \vec{o}_{n}=\left(\begin{array}{c}
o_{n 1} \\
o_{n \Delta}
\end{array}\right), \\
\vec{g}_{n}=\left(\begin{array}{c}
g_{n 1}-g_{N} \\
g_{n 2}-g_{n 1}
\end{array}\right) .
\end{array}
$$

The last equation shows how any noisy force applied to the spacecraft $\left(g_{N}\right)$ is only measured in the first channel (if there were no cross terms). On the other hand, the differential channel is sensitive to the difference of force noise applied to the first and the second test mass, $g_{1}$ and $g_{2}$ respectively.

The matrices read as

$$
\begin{aligned}
& \mathbf{D}=\left(\begin{array}{cc}
s^{2}+\omega_{1}^{2} & 0 \\
\omega_{2}^{2}-\omega_{1}^{2} & s^{2}+\omega_{2}^{2}
\end{array}\right), \\
& \mathbf{C}=\left(\begin{array}{cc}
G_{\mathrm{df}} H_{\mathrm{df}} & 0 \\
0 & G_{\text {sus }} H_{\mathrm{sus}}
\end{array}\right), \\
& \mathbf{S}=\left(\begin{array}{cc}
1 & 0 \\
\delta_{21} & 1
\end{array}\right),
\end{aligned}
$$

where $\omega_{1}$ and $\omega_{2}$ are the stiffness - the steady force gradient across the test mass housing per unit mass 12 coupling the motion of each test mass to the motion of the spacecraft; $G_{\mathrm{df}}$ and $G_{\text {sus }}$ are constant factors acting as calibration factors of the controller, $H_{\mathrm{df}}$ and $H_{\text {sus }}$. These are the control laws of the loop and will be considered known transfer functions in the following; $\delta_{21}$ is the interferometer cross-coupling, a small term accounting for the imperfection of the interferometer that will produce a spurious signal in the differential channel when only the first test mass moves. The interferometer has no coupling going from $o_{\Delta}$ to $o_{1}$ and therefore we set $\delta_{12}=0$ in the sensing matrix. The previous are the 5 parameters that we will consider in the following discussion, the ones characterizing the dynamics of the instrument.
TABLE I. Parameters for the LTP MDC2 model

\begin{tabular}{lccc}
\hline \hline \multicolumn{4}{c}{ Dynamical Parameters } \\
\hline Parameter & Value \\
\hline $\mathrm{G}_{\mathrm{df}}$ & 0.8 \\
$\mathrm{G}_{\text {sus }}$ & 1.15 \\
$\omega_{1}^{2}$ & $-11 \times 10^{-7}$ \\
$\omega_{2}^{2}$ & $-22 \times 10^{-7}$ \\
$\delta_{21}$ & $1.35 \times 10^{-4}$ \\
\hline \multicolumn{4}{c}{${ }_{5}$} \\
\hline Parameter & $o_{n 1} / o_{n}$ & $g_{n 1} / g_{n 2}$ & $g_{N}$ \\
\hline$p_{1}$ & $3.6 \times 10^{-12}$ & $7 \times 10^{-15}$ & $2.5 \times 10^{-10}$ \\
$p_{2}$ & $10 \times 10^{-3}$ & $5 \times 10^{-3}$ & $12 \times 10^{-3}$ \\
$p_{3}$ & 4.2 & 3 & 3.8 \\
$p_{4}$ & $1.8 \times 10^{-3}$ & $4 \times 10^{-4}$ & $1 \times 10^{-3}$ \\
$p_{5}$ & 8 & 8 & 8 \\
\hline \hline
\end{tabular}

The leading diagonal terms in Equation (3) describe the dynamics of each channel (for example, $s^{2}+\omega_{1}^{2}$ is Newton's law in the Laplace domain for the first test mass, with $\omega_{1}$ being the test mass stiffness), and the control law (for example, $G_{\mathrm{df}} H_{\mathrm{df}}$ stands for the dragfree transfer function controller on the first test mass, multiplied by a constant calibration factor, $\left.G_{\mathrm{df}}\right)$. The off-diagonal terms are the cross-couplings between the two channels appearing as triangles in Figure 1. From Equation (3) we can compute the response of the interferometer once all the dynamical and noise parameters are given as

$$
\vec{o}=\left(\mathbf{D} \cdot \mathbf{S}^{-1}+\mathbf{C}\right)^{-1}\left(-\mathbf{C} \vec{o}_{i}+\vec{g}_{n}+\mathbf{D} \cdot \mathbf{S}^{-1} \vec{o}_{n}\right) .
$$

This equation describes the interferometer output and will be the variable that we will use to evaluate the interferometer response. It may be useful to express the nominal output as a signal and two noise terms:

$$
\vec{o}=\mathbf{G}_{\mathbf{s}}(\Theta) \vec{o}_{i}+\mathbf{G}_{\mathbf{n o}}(\Theta) \vec{o}_{n}+\mathbf{G}_{\mathbf{n g}}(\Theta) \vec{g}_{n}
$$

where $\Theta=\left\{G_{\mathrm{df}}, G_{\mathrm{df}}, \omega_{1}^{2}, \omega_{2}^{2}, \delta_{21}\right\}$ are the unknown model parameters we are interested in determining.

Our model can be thought of as a first term which filters the input signal $\left(\vec{o}_{i}\right)$ and two further terms which filter the noise. It must be stated that, since our final aim is to characterize the noise model, the noise terms also contain information about our parameters. But, since we will be working in a high signal-to-noise ratio (SNR) regime, we will not consider this dependence in our analysis and we will further simplify the model with the approximations $\mathbf{G}_{\mathbf{n o}}(\omega, \Theta) \approx \mathbf{G}_{\mathbf{n o}}(\omega)$ and $\mathbf{G}_{\mathbf{n g}}(\omega, \Theta) \approx \mathbf{G}_{\mathbf{n g}}(\omega)$. This allows us to rewrite Equation (5) as

$$
\vec{o}=\mathbf{G}_{\mathbf{s}}(\Theta) \vec{o}_{i}+\vec{n}
$$

where $\vec{n}$ now represents the overall noise of the instrument. The first term then contains all the model dependence that we will be able to test with our experiments. 
The transfer function in this formulation now has the following components

$$
\begin{aligned}
G_{s}^{11} & =\frac{G_{\mathrm{df}} H_{\mathrm{df}}(\omega)}{\omega_{1}^{2}-\omega^{2}+G_{\mathrm{df}} H_{\mathrm{df}}(\omega)}, \\
G_{s}^{12} & =0, \\
G_{s}^{21} & =\frac{G_{\mathrm{df}} H_{\mathrm{df}}\left(\omega_{2}^{2}-\omega_{1}^{2}+\delta_{21}\left(\omega^{2}-\omega_{2}^{2}\right)\right)}{\left(\omega_{1}^{2}-\omega^{2}+G_{\mathrm{df}} H_{\mathrm{df}}\right)\left(\omega_{2}^{2}-\omega^{2}+G_{\mathrm{lfs}} H_{\mathrm{lfs}}\right)}, \\
G_{s}^{22} & =\frac{G_{\mathrm{lfs}} H_{\mathrm{lfs}}(\omega)}{\omega_{2}^{2}-\omega^{2}+G_{\mathrm{lfs}} H_{\mathrm{lfs}}(\omega)},
\end{aligned}
$$

where we can see that by injecting and measuring in the same channel (i.e., testing the diagonal terms), we are able to determine either $\left\{G_{\mathrm{df}}, \omega_{1}^{2}\right\}$ or $\left\{G_{\text {sus }}, \omega_{2}^{2}\right\}$, and it is through the non-diagonal (cross-coupling) term that we can determine the $\delta_{21}$ parameter and the difference between stiffnesses, $\omega_{2}^{2}-\omega_{1}^{2}$. The experiments in this MDC were designed to test these possible combinations of injected signals, as described in the following.

\section{B. Model parameters}

Our model is defined by a total of 30 parameters, which can be divided into two groups: noise parameters and dynamical parameters. The first ones are those ones used to set the noise shapes of the individual noise contributions - force noise $\vec{g}_{n}$ and interferometer read-out noise $\vec{o}_{n}$ in Equation (5) — that will set the final instrument noise level. Each contribution is described as

$$
S(\omega)=p_{1}^{2}\left(1+\frac{1}{\left(\frac{\omega}{2 \pi p_{2}}\right)^{p_{3}}}+\frac{1}{\left(\frac{\omega}{2 \pi p_{4}}\right)^{p_{5}}}\right)^{1 / 2},
$$

and therefore 5 parameters are required for each of them, for a total of 25 to describe all noise contributions. We need to add to these the 5 parameters that characterize the joint dynamical behavior of the spacecraft and test masses. Only the latter will be the parameters that we will be interested in recovering from the data in this challenge. As stated above these are: stiffness for each test mass $\left(\omega_{1}^{2}, \omega_{2}^{2}\right)$, calibration for each controller $\left(G_{\mathrm{df}}, G_{\mathrm{sus}}\right)$ and interferometer cross-coupling $\left(\delta_{21}\right)$.

Table【contains all numerical values used in the second Mock Data Challenge, and therefore fully characterizes the model. Although the model allows for different noise levels for $x_{1}$ and $x_{\Delta}$ interferometer noise, we did not use this degree of freedom and set both interferometers to behave equally. The same applies to the force noise acting on both test masses.

\section{Experiments}

Three experiments were proposed for MDC2. These were originally motivated by first studies about the sensitivity attainable by injected signals during the mis-

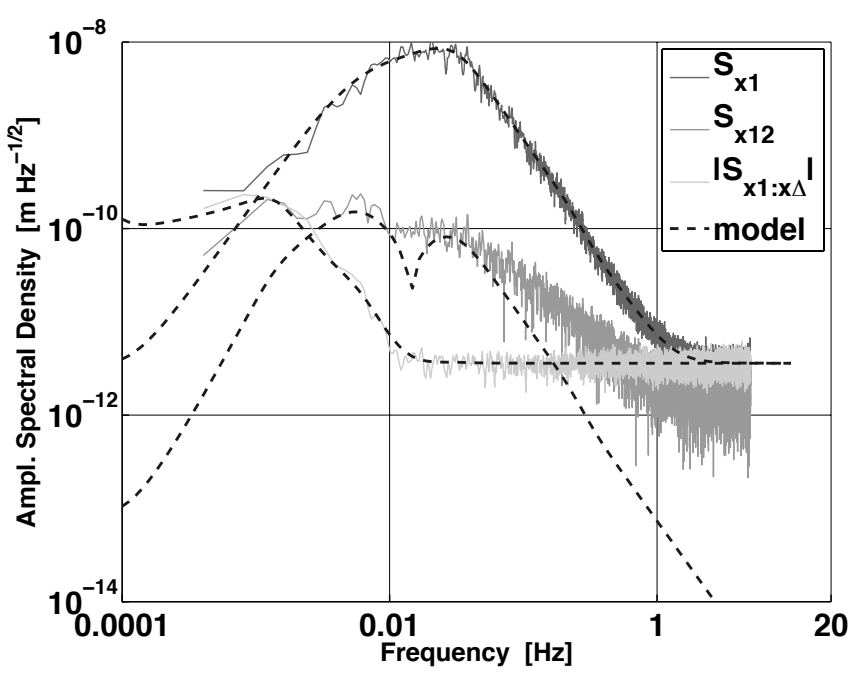

FIG. 2. Amplitude spectral density of a noise realization of the LTP MDC2 noise model compared to analytical curves. We compare the noise of the first channel $\left(S_{\mathrm{x} 1}\right)$, the second channel $\left(S_{\mathrm{x} \Delta}\right)$ and the absolute value of the cross-spectra between both $\left(S_{\mathrm{x} 1: \mathrm{x} \Delta}\right)$.

sion [13] and correspond to a frequency sweep in the measurement bandwidth at four different frequencies. Our experiments in MDC2 consider only the possibility of injected signals as simulated interferometric signals, the so-called interferometric bias, which we have labelled in Equation (5) and in Figure 1 as $\vec{o}_{i}$. LISA Pathfinder will allow other kinds of injected signals, for instance, forces applied to the spacecraft via the thrusters or forces directly applied to the test masses via the capacitive sensors but, as stated above, it is not the aim of this work to explore all capabilities of the mission. In that sense, extending the analysis to include all possible injection signals is one of the aims of the forthcoming LISA Pathfinder MDCs. The three proposed experiments for this challenge were the following:

Experiment 1: Two signals are injected independently into the first and second channel. Each signal is a sequence of sinusoids with different amplitudes, frequencies and duration, all of them known to the data analysis team. This experiment is the richer in terms of frequencies injected to the system, and the one with best expected parameter estimates, as we show in the following section.

Experiment 2: A signal is injected in the first channel and both test mass stiffnesses are set to the same value, different than the value for the two other experiments. This configuration represents the matched stiffness configuration in the real LISA Pathfinder satellite. This state can be achieved by commanding an equal bias voltage on the electrodes of the inertial sensors at a level which dominates all other stiffness effects thus resulting in an 

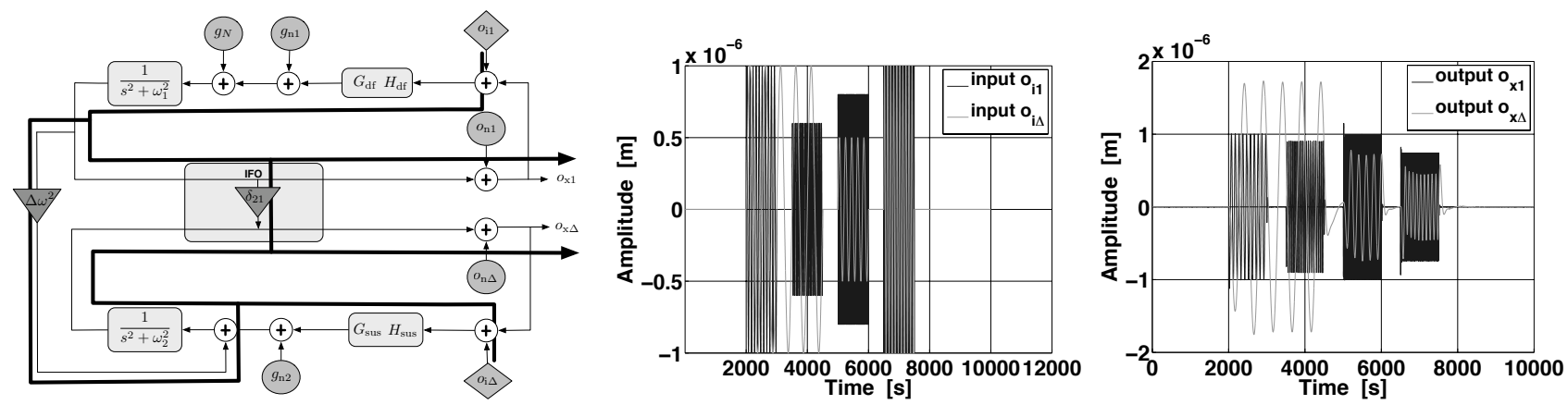

Experiment 2
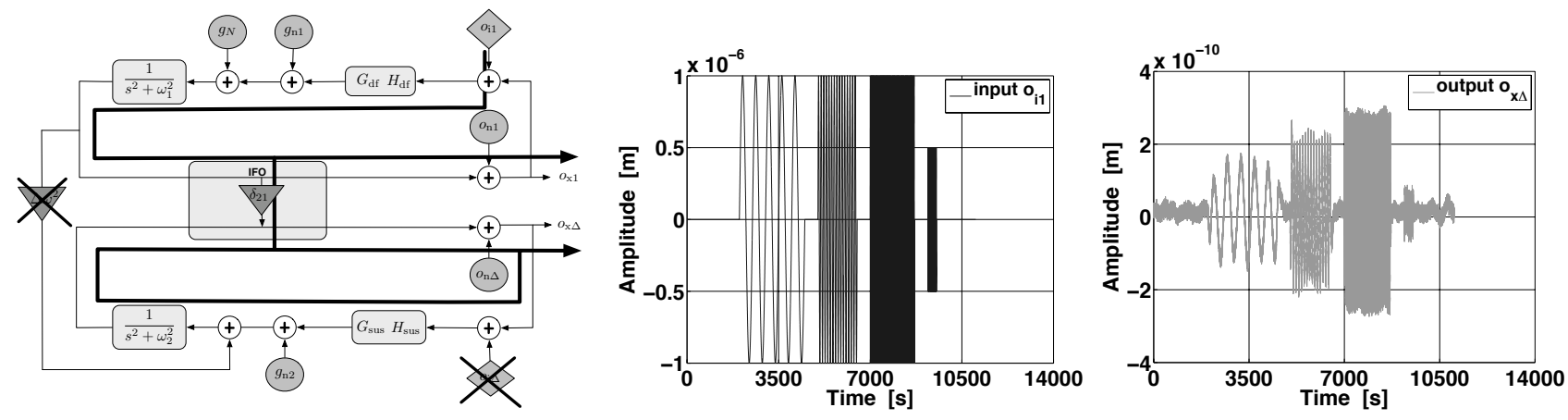

Experiment 3
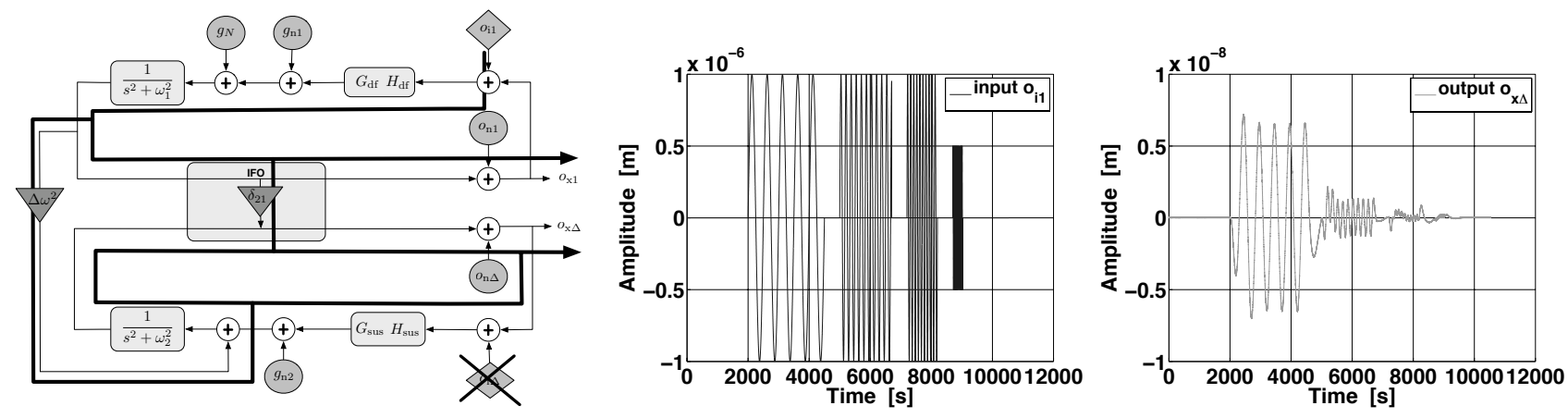

FIG. 3. The three MDC2 experiments. From left to right: scheme of injected signal, input signal and output signal. From top to bottom: experiment 1, 2 and 3. Only $x_{12}$ output is shown for experiments 2 and 3 , the response of the first channel to the injected signal is similar to the one shown in experiment 1.

equal coupling between the two test masses and the spacecraft. This scheme is particularly useful since it would ideally decouple any external force from the differential measurement. However, in our simplified model there is already a second crossterm, the interferometer cross-coupling, $\delta_{21}$, mixing both channels - see Equation (9). Being the only remaining cross-coupling in this experiment, this parameter should therefore be obtained with the greatest accuracy when analyzing this data set.

Experiment 3: The last experiment again applies only one signal to the first channel but without matching the stiffness for both test masses. This exper- iment tests the ability to recover the same parameters that we determine in Experiment 1, but by only injecting signals into the first channel.

The data set in MDC2 also included a run without any injected signal from where the instrument performance could be evaluated. A typical noise realisation for this model is shown in Figure 2 whereas the three MDC2 experiments are represented in Figure 3, all of them generated using LTPDA methods. The concept behind the data generation process is to translate the transfer functions appearing in Equation (5) into digital filters, and then use those filters to translate the input signal into the measured output. Since the measured data is a combi- 
nation of signal and noise, the data generation procedure is consequently split into two branches that are added at the end. The generation of the signal part is straightforward since it only requires the filtering of a deterministic signal. In contrast, the noise part requires the use of digital filters to color white-noise and to do it in such a way that the noise cross-correlation properties between the two channels are correctly reproduced. A detailed description of this process can be found in [14.

\section{DATA ANALYSIS}

\section{A. Bayesian estimation}

We would now like to infer unknown parameters from the simulated data. To this end we need to derive the posterior probability distribution of the parameters, that is, the conditional probability distribution of the parameters for the given data at hand. The posterior distribution expresses the information about the parameters by assigning probabilities across parameter space, and by that allows us to derive the most likely values and their uncertainties [15, 16]. The posterior distribution is given by Bayes' theorem, and it depends on the data as well as any other prior information $I$ :

$\mathrm{P}(\Theta \mid D, I)=\frac{\mathrm{P}(\Theta \mid I) \times \mathrm{P}(D \mid \Theta, I)}{\mathrm{P}(D \mid I)} \propto \mathrm{P}(\Theta \mid I) \times \mathrm{P}(D \mid \Theta, I)$.

The prior probability distribution $\mathrm{P}(\Theta \mid I)$ expresses information we may have about the parameter values (in addition to the data $D$ ), while the likelihood function $\mathrm{P}(D \mid \Theta, I)$ describes the probabilistic relationship between parameters and the (noisy) measurements. The evidence $\mathrm{P}(D \mid I)$ is usually not of concern for parameter estimation purposes and constitutes a normalizing constant here. In this work we will assume uniform prior distributions for all parameters, i.e., the prior density $\mathrm{P}(\Theta \mid I)$ is constant across the allowed region as defined in Table $\mathrm{I}$.

Given the simplified model in Equation (6) we start by assuming that the noise term $\vec{n}$ is Gaussian. The noise in each of the two output channels is characterized by the (known) one-sided power spectral density functions $S_{\mathrm{x} 1}(f)$ and $S_{\mathrm{x} \Delta}(f)$, respectively. In addition, the noise is assumed to be correlated between the two outputs, which is expressed through the cross spectral density $S_{\mathrm{x} 1: \mathrm{x} \Delta}(f)$. Due to the colored noise it will be convenient to express the likelihood function in terms of the Fourier transformed data. The likelihood function then is given by

$$
\begin{aligned}
& p(D \mid \Theta, I)=\left[(2 \pi)^{N / 2} \operatorname{det} \boldsymbol{\Sigma}\right]^{-1 / 2} \\
& \times \exp \left[-\frac{1}{2}\left(\vec{o}-\mathbf{G}_{\mathbf{s}}(\Theta) \vec{o}_{i}\right)^{T} \boldsymbol{\Sigma}^{-1}\left(\vec{o}-\mathbf{G}_{\mathbf{s}}(\Theta) \vec{o}_{i}\right)\right]
\end{aligned}
$$

where $\mathbf{J}(\Theta)$ is the Fisher information matrix. For our particular case it will shown to be useful to use the Cramér-Rao bound expressed as [18,

so that (up to a multiplicative factor) the logarithmic likelihood is proportional to the quadratic form

$$
\log (p(D \mid \Theta, I)) \propto-\frac{1}{2}\left(\vec{o}-\mathbf{G}_{\mathbf{s}}(\Theta) \vec{o}_{i}\right)^{T} \boldsymbol{\Sigma}^{-1}\left(\vec{o}-\mathbf{G}_{\mathbf{s}}(\Theta) \vec{o}_{i}\right),
$$

where $\boldsymbol{\Sigma}$ is the covariance matrix of the (Fourier domain) noise term $\vec{n}$. The covariance matrix entries are then defined by the spectral and cross-spectral density values corresponding to the Fourier frequencies. Most of $\Sigma$ 's entries are zero (since only the terms corresponding to the same Fourier frequency are correlated) and the quadratic form may be rearranged so that $\boldsymbol{\Sigma}$ is of a block-diagonal form and the likelihood expression simplifies to a sum over the blocks of correlated terms at each frequency bin:

$$
\log (p(D \mid \Theta, I)) \propto-\frac{1}{2} \sum_{j} \operatorname{Re}\left(r_{j}^{T} \Sigma_{j}^{-1} r_{j}\right)
$$

where $j=0, \ldots, N / 2$ is an index over the Fourier frequencies $f_{j}$, and $r_{j}$ and $\Sigma_{j}$ denote the two (complexvalued) residual terms and corresponding covariance matrix at frequency $f_{j}$ :

$$
\begin{aligned}
r_{j} & =\left(\begin{array}{c}
{\left[o_{\mathrm{x} 1}-\left(G_{11}(\Theta) o_{\mathrm{i} 1}+G_{12}(\Theta) o_{\mathrm{i} \Delta}\right)\right]\left(f_{j}\right)} \\
{\left[o_{\mathrm{x} \Delta}-\left(G_{21}(\Theta) o_{\mathrm{i} 1}+G_{22}(\Theta) o_{\mathrm{i} \Delta}\right)\right]\left(f_{j}\right)}
\end{array}\right), \\
\Sigma_{j} & =\frac{N}{4 \Delta_{t}}\left(\begin{array}{cc}
S_{\mathrm{x} 1}\left(f_{j}\right) & S_{\mathrm{x} 1: \mathrm{x} \Delta}\left(f_{j}\right)^{*} \\
S_{\mathrm{x} 1: \mathrm{x} \Delta}\left(f_{j}\right) & S_{\mathrm{x} \Delta}\left(f_{j}\right)
\end{array}\right) .
\end{aligned}
$$

\section{B. Optimal parameter estimation errors}

In order to get an idea of what kind of information the simulated experiments will provide, we will use the Fisher information formalism to estimate the measurement errors to be expected from the different experimental settings. The Fisher information and the corresponding Cramér-Rao bound (CRB) provide an estimate of the measurement uncertainties to be expected in the limit of a large signal-to-noise ratio (SNR) [17]. For an unbiased estimate of $\Theta$, the $\mathrm{CRB}$ can be expressed as

$$
\operatorname{cov}(\Theta) \geq \mathbf{J}^{-1}(\Theta),
$$




$$
[\mathbf{J}(\Theta)]_{l m}=\sum_{j, k}\left[\frac{1}{2 \pi} \int_{-\infty}^{\infty} d \omega \frac{1}{S_{j k}(\omega, \Theta)} \frac{\partial o_{j}(\omega, \Theta)}{\partial \theta_{l}} \frac{\partial o_{k}(\omega, \Theta)}{\partial \theta_{m}}+\frac{T}{4 \pi} \int_{-\infty}^{\infty} d \omega \frac{1}{S_{j k}^{2}(\omega, \Theta)} \frac{\partial S_{j k}(\omega, \Theta)}{\partial \theta_{l}} \frac{\partial S_{j k}(\omega, \Theta)}{\partial \theta_{m}}\right]
$$

where we sum over the two channels; $o_{x 1}$ and $o_{x \Delta}$ being the two components of the nominal output, $S_{j k}(\omega, \boldsymbol{\Theta})$ the components of the cross-spectrum matrix and $T$ the integration time. We are considering here the parametric dependence of the noise terms - Equation (5). Although we will drop it in the next step, we want to explicitly state that term since it is usually not considered in the Fisher matrix analysis among the gravitational wave community [17, but it may turn out to be relevant in future analysis since the noise model characterization is the final purpose of the LTP mock data challenges. However, for this first application, and to avoid cumbersome equations, we decided not to include those terms considering that they will not introduce any relevant information in the high SNR regime where we are working. Switching therefore to Equation (6) and substituting into Equation (18) leads to

$$
\begin{aligned}
& {[\mathbf{J}(\Theta)]_{l m} } \\
= & \sum_{j, k} \frac{o_{i, j} o_{i, k}^{*}}{2 \pi} \int_{-\infty}^{\infty} d \omega \frac{1}{S_{j k}(\omega)} \frac{\partial G_{j k}(\Theta)}{\partial \theta_{l}} \frac{\partial G_{j k}(\Theta)}{\partial \theta_{m}},
\end{aligned}
$$

where now $o_{i, 1}$ and $o_{i, \Delta}$ are the two components of the input signal and $G_{j k}(\boldsymbol{\Theta})$ the components of the transfer function. We will use Equation $(19)$ in the following to evaluate the CRB in each experiment. It is important to keep in mind that the three experiments analyzed here contain different configurations of the instrument, meaning that both the transfer function elements and the signals are changing in each experiment.

TABLE II. Crámer-Rao bound. Values between parenthesis expressed in relative parts per thousand (\%)

\begin{tabular}{c|ccc}
\hline \hline Parameter & Exp. 1 & Exp. 2 & Exp. 3 \\
\hline$\sigma_{G_{\mathrm{df}}}$ & $2 \times 10^{-5}(0.02)$ & $5 \times 10^{-5}(0.06)$ & $2 \times 10^{-4}(0.2)$ \\
$\sigma_{G_{\text {sus }}}$ & $3 \times 10^{-7}(0.0002)$ & $3 \times 10^{-3}(3)$ & $3 \times 10^{-4}(0.3)$ \\
$\sigma_{\omega_{1}}$ & $6 \times 10^{-10}(0.5)$ & $3 \times 10^{-6}(1000)$ & $9 \times 10^{-8}(80)$ \\
$\sigma_{\omega_{2}}$ & $3 \times 10^{-10}(0.1)$ & $3 \times 10^{-6}(1000)$ & $9 \times 10^{-8}(40)$ \\
$\sigma_{\delta_{21}}$ & $6 \times 10^{-8}(0.5)$ & $4 \times 10^{-8}(0.2)$ & $1 \times 10^{-7}(0.9)$ \\
$\sigma_{\Delta \omega}$ & $5 \times 10^{-10}(0.4)$ & $6 \times 10^{-10}(-)$ & $3 \times 10^{-10}(0.3)$ \\
\hline \hline
\end{tabular}

Table III summarizes the optimal error estimates that the data analysis should return. The last column refers to the achievable standard deviation in the difference between squared stiffnesses, $\Delta \omega^{2}=\omega_{2}^{2}-\omega_{1}^{2}$. This will be only indirectly estimated by the analysis, but we added it to the table, firstly, because the cross-coupling between both channels depends directly on this difference, but also because the error in the estimation of the stiffnesses difference depends on the non-diagonal terms of the co- variance matrix. This quantity adds then some more information not contained in the other parameters, which are extracted purely from the diagonal terms. The $\sigma_{\Delta \omega}$ error is computed as

$$
\sigma_{\Delta \omega}^{2}=\sigma_{\omega_{1}}^{2}+\sigma_{\omega_{2}}^{2}-2 \sigma_{\omega_{1}, \omega_{2}}
$$

where $\sigma_{\omega_{1}}^{2}$ and $\sigma_{\omega_{1}}^{2}$ are the variances of the stiffness squared of test mass 1 and test mass 2 , and $\sigma_{\omega_{1}, \omega_{2}}$ is the covariance term containing the correlation between both stiffnesses. A remarkable result from this analysis is that a single experiment injecting a signal in both channels (experiment 1) is enough to determine all parameters with high precision. In fact, this experiment is preferable to the other experiments which only inject signals in the $x_{1}$ channel. Only the matched stiffness experiment (experiment 2) gives a slightly better estimation of the interferometer cross-coupling. Precision in this parameter is gained however at expenses of increasing the uncertainty in the determination of the absolute value of the stiffnesses, reaching in this case $100 \%$. In principle, if we take into account our simplified model, experiment 3 would be redundant, not adding more information (apart from statistical averaging) than what we get from experiments 1 and 2 .

In order to give some more insight in what refers the difference between experiments we provide in Table III the correlation matrices as computed with the previous formalism. These results complement the ones in Table [I] since the diagonal terms of the latter correspond to the values reported in the former. Comparison between correlation matrices show how experiment 1 is disentangling the different parameteres dependences more efficiently. In particular, it is the only experiment which is able to differentiate the contribution of the two stiffnesses. The reason for that being that it is the only experiment with a signal injected in the differential channel.

\section{Combining the results of experiments}

\section{The information propagation problem}

As opposed to the usual application of Bayesian parameter estimation in LISA, where a single set of data is used to determine the parameters of a multiplicity of systems, i.e., astrophysical sources, in our case we use different sets of data (experiments) to characterize a unique system, the LTP experiment. Thus, once we have obtained the parameter estimates for each experiment we still need to go further to achieve our final goal. Since each experiment can be adding valuable, but partial, information about the instrument, we need to find a scheme 
TABLE III. Correlation matrices for MDC2 experiments

\begin{tabular}{|c|c|c|c|c|c|}
\hline & $\mathrm{G}_{\mathrm{df}}$ & $\mathrm{G}_{\text {sus }}$ & $\omega_{1}^{2}$ & $\omega_{2}^{2}$ & $\delta_{21}$ \\
\hline & \multicolumn{5}{|c|}{ Experiment 1} \\
\hline $\mathrm{G}_{\mathrm{df}}$ & 1 & 0.0003 & -0.1 & -0.001 & -0.2 \\
\hline $\mathrm{G}_{\text {sus }}$ & 0.0003 & 1 & -0.3 & -0.5 & -0.001 \\
\hline$\omega_{1}^{2}$ & -0.1 & -0.3 & 1 & 0.5 & 0.5 \\
\hline$\omega_{2}^{2}$ & -0.001 & -0.5 & 0.5 & 1 & 0.005 \\
\hline \multirow[t]{2}{*}{$\delta_{21}$} & -0.2 & -0.001 & 0.5 & 0.005 & 1 \\
\hline & \multicolumn{5}{|c|}{ Experiment 2} \\
\hline $\mathrm{G}_{\mathrm{df}}$ & 1 & 0.4 & -0.6 & -0.6 & 0.2 \\
\hline $\mathrm{G}_{\text {sus }}$ & 0.4 & 1 & -0.7 & -0.7 & 0.3 \\
\hline$\omega_{1}^{2}$ & -0.6 & -0.7 & 1 & $\approx 1$ & -0.4 \\
\hline$\omega_{2}^{2}$ & -0.6 & -0.7 & $\approx 1$ & 1 & -0.4 \\
\hline \multirow[t]{2}{*}{$\delta_{21}$} & 0.2 & 0.3 & -0.4 & -0.4 & 1 \\
\hline & \multicolumn{5}{|c|}{ Experiment 3} \\
\hline $\mathrm{G}_{\mathrm{df}}$ & 1 & 0.03 & -0.02 & -0.02 & 0.04 \\
\hline $\mathrm{G}_{\text {sus }}$ & 0.03 & 1 & -0.8 & -0.8 & 0.3 \\
\hline$\omega_{1}^{2}$ & -0.02 & -0.8 & 1 & $\approx 1$ & -0.09 \\
\hline$\omega_{2}^{2}$ & -0.02 & -0.8 & $\approx 1$ & 1 & -0.09 \\
\hline$\delta_{21}$ & 0.04 & 0.3 & -0.09 & -0.09 & 1 \\
\hline
\end{tabular}

that allows us to include all the information in a final set of parameters.

The efficient combination of results is also an important problem to solve in terms of mission operations. It should be noted that the LISA Pathfinder mission will be a space laboratory with approximately 100 channels being sampled and more than 50 parameters defining its performance. It will therefore be crucial to combine the results from one experiment with the ones following. For instance, we may be interested in using the determination of the stiffness to calibrate the thrusters in a forthcoming experiment. Given the limited mission time and the high numbers of experiments to be performed, the need for a clear combination scheme is evident. We explore in the following how to take advantage of the posterior distribution to that end.

\section{The general case}

a. Identical parameter sets First consider the case where the parameter sets are identical for the data sets to be combined (as e.g. in Experiments 1 and 3 above). Suppose we have a parameter vector $\Theta$ and two data sets $D_{1}$ and $D_{2}$. Similar to the general case in Equation (12), the posterior distribution $\mathrm{P}\left(\Theta \mid D_{1}, D_{2}, I\right)$ is then given by

$$
\mathrm{P}\left(\Theta \mid D_{1}, D_{2}, I\right) \propto \underbrace{\overbrace{\mathrm{P}(\Theta \mid I)}^{\text {prior }} \times \overbrace{\mathrm{P}\left(D_{1} \mid \Theta, I\right)}^{\text {likelihood }} \times \underbrace{\mathrm{P}\left(D_{2} \mid \Theta, I\right)}_{\text {likelihood }}}_{\text {prior }},
$$

where the same expression may be motivated by either taking the likelihood to be the product of the individual experiments' likelihoods or by analyzing the experiments one after the other and using the posterior from the first experiment as the prior for the second experiment (21).

b. Differing parameter sets In order to deal with differing parameter sets that only partially overlap, one needs to consider the union of all the unknowns as the set of parameters. Combining data from different experiments then works exactly as in Equation (21), only that the parameter vector $\Theta$ is now the extended parameter set. The likelihood functions are exactly the same as in the individual-experiment case, with the only difference that, as functions of the extended parameter set, they do not depend on some of the parameters.

Consider the case where two data sets $D_{1}$ and $D_{2}$ depend on parameter $\vartheta_{1}$, while the parameters $\vartheta_{2}$ and $\vartheta_{3}$ are specific for $D_{1}$ and $D_{2}$, respectively. Assuming the error terms for both experiments to be independent, the joint likelihood function then is the product

$$
\begin{aligned}
& \mathrm{P}\left(D_{1}, D_{2} \mid \vartheta_{1}, \vartheta_{2}, \vartheta_{3}, I\right) \\
= & \mathrm{P}\left(D_{1} \mid \vartheta_{1}, \vartheta_{2}, \vartheta_{3}, I\right) \times \mathrm{P}\left(D_{2} \mid \vartheta_{1}, \vartheta_{2}, \vartheta_{3}, I\right), \\
= & \mathrm{P}\left(D_{1} \mid \vartheta_{1}, \vartheta_{2}, I\right) \times \mathrm{P}\left(D_{2} \mid \vartheta_{1}, \vartheta_{3}, I\right) .
\end{aligned}
$$

In order to simplify things, in the following we will introduce the assumption that the conditional prior $\mathrm{P}\left(\vartheta_{2} \mid \vartheta_{1}, \vartheta_{3}, I\right)$ is independent of $\vartheta_{3}$, i.e.,

$$
\mathrm{P}\left(\vartheta_{2} \mid \vartheta_{1}, \vartheta_{3}, I\right)=\mathrm{P}\left(\vartheta_{2} \mid \vartheta_{1}, I\right)
$$

(since $\vartheta_{2}$ and $\vartheta_{3}$ were the parameters which did not jointly affect both experiments, this may be easily satisfied, for example if $\mathrm{P}\left(\vartheta_{1}, \vartheta_{2}, \vartheta_{3} \mid I\right)=\mathrm{P}\left(\vartheta_{1} \mid I\right) \times \mathrm{P}\left(\vartheta_{2} \mid I\right) \times$ $\left.\mathrm{P}\left(\vartheta_{3} \mid I\right)\right)$. When considering additional data $D_{2}$, the change in the (marginal) posterior distribution of the two parameters $\vartheta_{1}$ and $\vartheta_{2}$ then is given by

$$
\mathrm{P}\left(\vartheta_{1}, \vartheta_{2} \mid D_{1}, D_{2}, I\right)=\mathrm{P}\left(\vartheta_{1}, \vartheta_{2} \mid D_{1}, I\right) \times \frac{\mathrm{P}\left(\vartheta_{1} \mid D_{2}, I\right)}{\mathrm{P}\left(\vartheta_{1} \mid I\right)}
$$

so that in order to "update" the posterior distribution of $\vartheta_{1}$ and $\vartheta_{2}$ using the data $D_{2}$ that depends on the additional parameter $\vartheta_{3}$, we only need to consider the marginal prior and posterior distributions of the common parameter $\vartheta_{1}, \mathrm{P}\left(\vartheta_{1} \mid I\right)$ and $\mathrm{P}\left(\vartheta_{1} \mid D_{2}, I\right)$. We can see that when updating the posterior by another posterior (24), the (marginal) prior needs to be cancelled out, otherwise it would enter twice into the resulting posterior. Since by combining the posteriors we will only learn about the common parameter $\vartheta_{1}$ here, it will be easier to also integrate out $\vartheta_{2}$ and only consider the (marginal) distributions involving $\vartheta_{1}$, which then leads to

$$
\mathrm{P}\left(\vartheta_{1} \mid D_{1}, D_{2}, I\right)=\mathrm{P}\left(\vartheta_{1} \mid D_{1}, I\right) \times \frac{\mathrm{P}\left(\vartheta_{1} \mid D_{2}, I\right)}{\mathrm{P}\left(\vartheta_{1} \mid I\right)} .
$$

The higher-dimensional case works completely analogously, just by considering the parameters $\vartheta_{1}, \vartheta_{2}, \vartheta_{3}$ to be sub-vectors. 


\section{The Gaussian approximation}

As we will see below, the derived posterior distributions often turn out to be well approximated by a multivariate Gaussian distribution with mean $\mu$ and covariance matrix $\boldsymbol{\Sigma}$ :

$$
\begin{aligned}
& p(\mathbf{x} \mid D) \approx p(\mathbf{x} ; \mu, \mathbf{\Sigma}) \\
= & \frac{1}{(2 \pi)^{N}|\Sigma|^{1 / 2}} \exp \left\{-\frac{1}{2}(\mathbf{x}-\mu)^{T} \mathbf{\Sigma}^{-\mathbf{1}}(\mathbf{x}-\mu)\right\} .
\end{aligned}
$$

If the posterior distributions are expressed as Gaussians, it is particularly easy to analytically propagate prior and posterior information as described in the previous subsection; in the following we will therefore apply these results to the Gaussian case. As a further simplification, we will also assume all prior distributions to be uniform.

a. Identical parameter sets In order to combine the results coming from two experiments $D_{1}$ and $D_{2}$, we will need to combine their two posterior distributions as in Equation (21). The results from experiments $D_{1}$ and $D_{2}$ will be summarized by parameters' posterior means and covariances $\left\{\mu_{\mathbf{1}}, \boldsymbol{\Sigma}_{\mathbf{1}}\right\}$ and $\left\{\mu_{\mathbf{2}}, \boldsymbol{\Sigma}_{\mathbf{2}}\right\}$, respectively. Assuming uniform priors, we can now combine both as

$$
\begin{aligned}
p\left(\mathbf{x} \mid D_{1}, D_{2}\right) & =p\left(\mathbf{x} \mid D_{1}\right) \times p\left(\mathbf{x} \mid D_{2}\right) \\
& =p\left(\mathbf{x} ; \mu_{\mathbf{1}}, \boldsymbol{\Sigma}_{\mathbf{1}}\right) \times p\left(\mathbf{x} ; \mu_{\mathbf{2}}, \mathbf{\Sigma}_{\mathbf{2}}\right) \\
& =p\left(\mathbf{x} ; \mu_{\mathbf{c}}, \mathbf{\Sigma}_{\mathbf{c}}\right),
\end{aligned}
$$

i.e., the product of posterior densities again is Gaussian with mean $\mu_{\mathbf{c}}$ and covariance $\boldsymbol{\Sigma}_{\mathbf{c}}$. The parameters of the combined posterior may then be derived using the following relationship

$$
\begin{aligned}
& (\mathbf{x}-\mathbf{u})^{\mathbf{T}} \mathbf{U}^{-\mathbf{1}}(\mathbf{x}-\mathbf{u})+(\mathbf{x}-\mathbf{v})^{\mathbf{T}} \mathbf{V}^{-\mathbf{1}}(\mathbf{x}-\mathbf{v}), \\
= & (\mathbf{x}-\mathbf{w})^{\mathbf{T}} \mathbf{W}^{-\mathbf{1}}(\mathbf{x}-\mathbf{w}),
\end{aligned}
$$

where

$$
\mathbf{w}=\mathbf{W}^{-1}[\mathbf{U u}+\mathbf{V v}], \quad \mathbf{W}=\mathbf{U}+\mathbf{V},
$$

so that the new mean and covariance turn out as

$$
\begin{aligned}
\Sigma_{\mathrm{c}}^{-1} & =\Sigma_{1}^{-1}+\Sigma_{2}^{-1} \\
\mu_{\mathrm{c}} & =\Sigma_{\mathrm{c}}\left[\Sigma_{1}^{-1} \mu_{1}+\Sigma_{2}^{-1} \mu_{2}\right]
\end{aligned}
$$

[15]. The same argument is easily extended to an arbitrary number $N$ of experiments as

$$
\begin{aligned}
\boldsymbol{\Sigma}_{\mathbf{N}}^{-\mathbf{1}} & =\sum_{i=1}^{N} \boldsymbol{\Sigma}_{i}^{-1} \\
\mu_{\mathbf{N}} & =\boldsymbol{\Sigma}_{\mathbf{N}} \sum_{i=1}^{N} \boldsymbol{\Sigma}_{i}^{-1} \mu_{i} .
\end{aligned}
$$

b. Differing parameter sets Now suppose we have results of two experiments in which the parameter sets were not quite identical, as in the previous Section IIIC $2 \mathrm{~b}$. One may now either directly derive estimates of the marginal distribution (i.e., their means and covariances) and use those to combine the marginal posteriors as in Equation (25) and in the previous section. Otherwise, if given only the joint distributions (means and covariances) of the differing (but intersecting) parameter sets, these may also be marginalized analytically. For a Gaussian distribution the marginal distribution of a subset of the variables is simply given by the corresponding subset of mean and covariance parameters, i.e., by dropping the rows and columns corresponding to the variables that are integrated out.

\section{Implementation}

Our implementation follows a four-step procedure to analyze each experiment, all of them implemented as LTPDA methods. The first step is to Fourier transform the data. The noise's power spectral density is estimated using the Welch method [19] and applying a BlackmanHarris window. We can then compute the log-likelihood (13) and therefore find the maximum of the posterior density function using a (Nelder-Mead) simplex search algorithm [20. Since with our strong signal injections the likelihood surface apparently does not tend to exhibit many secondary maxima, this step is usually sufficient to determine the parameters to good accuracy and it is also more efficient than waiting for the Metropolis sampler to converge. However, if the likelihood surface shows secondary maxima, this method may lead to an erroneous result. Next, the posterior covariance among parameters according to input signals, noise and the relevant transfer functions, is estimated by numerically evaluating the Fisher information matrix at the maximum determined in the previous optimization step. And finally, we can integrate the posterior using a Markov Chain Monte Carlo (MCMC) approach. We use a Metropolis algorithm [15, 21 that will generate random samples from the parameters' (5-dimensional) posterior distribution. Generation of these samples is relatively easy based only on the expression of the (unnormalized) posterior density function (12) or (14)).

In order to enhance convergence of the MCMC sampler, we apply tempering to the posterior density function, which is supposed to make it more tractable and keep the algorithm from getting stuck in local optima. In the MCMC context, tempering is commonly implemented by applying an exponent to the probability density to be sampled from, i.e., instead of using the posterior $p(\theta \mid D, I)$, the tempered posterior $p(\theta \mid D, I)^{\frac{1}{T}}$ is considered, where $T \geq 1$ is the "temperature" 21, 22. The $\frac{1}{T}$ exponent smoothens the targeted density function, which generally allows the sampler to move more quickly and widely through parameter space and to traverse between local modes more easily. The following expression describes the temperature profile used in our 
TABLE IV. Estimated parameters for independent experiments.

\begin{tabular}{|c|c|c|c|c|}
\hline Param. & Value $\theta$ & Estimated $\hat{\theta} \pm \sigma$ & $|\theta-\hat{\theta}| / \sigma$ & $\sigma / \sigma_{\mathrm{CRB}}$ \\
\hline & \multicolumn{4}{|c|}{ Experiment 1} \\
\hline $\mathrm{G}_{\mathrm{df}}$ & 0.8 & $0.80002 \pm 0.00002$ & 1.0 & 1.0 \\
\hline $\mathrm{G}_{\text {sus }}$ & 1.15 & $1.1500001 \pm 0.0000003$ & 0.4 & 0.9 \\
\hline$\omega_{1}^{2}$ & $-1.1 \times 10^{-6}$ & $(-1.0991 \pm 0.0005) \times 10^{-6}$ & 1.7 & 1.0 \\
\hline$\omega_{2}^{2}$ & $-2.2 \times 10^{-6}$ & $(-2.2001 \pm 0.0003) \times 10^{-6}$ & 0.3 & 1.0 \\
\hline$\delta_{21}$ & $1.35 \times 10^{-4}$ & $(1.3502 \pm 0.0006) \times 10^{-4}$ & 0.3 & 1.0 \\
\hline \multirow[t]{2}{*}{$\Delta \omega^{2}$} & $-1.1 \times 10^{-6}$ & $(-1.1010 \pm 0.0005) \times 10^{-6}$ & 2.1 & 1.0 \\
\hline & \multicolumn{4}{|c|}{ Experiment 2} \\
\hline $\mathrm{G}_{\mathrm{df}}$ & 0.8 & $0.80011 \pm 0.00005$ & 2.2 & 1.0 \\
\hline $\mathrm{G}_{\text {sus }}$ & 1.15 & $1.147 \pm 0.004$ & 0.8 & 1.0 \\
\hline$\omega_{1}^{2}$ & $-2.4 \times 10^{-6}$ & $(-5 \pm 3) \times 10^{-6}$ & 0.8 & 1.0 \\
\hline$\omega_{2}^{2}$ & $-2.4 \times 10^{-6}$ & $(-5 \pm 3) \times 10^{-6}$ & 0.8 & 1.0 \\
\hline$\delta_{21}$ & $1.35 \times 10^{-4}$ & $(1.3497 \pm 0.0003) \times 10^{-4}$ & 1.0 & 0.9 \\
\hline \multirow[t]{2}{*}{$\Delta \omega^{2}$} & 0 & $(-3 \pm 6) \times 10^{-10}$ & 0.5 & 1.1 \\
\hline & \multicolumn{4}{|c|}{ Experiment 3} \\
\hline $\mathrm{G}_{\mathrm{df}}$ & 0.8 & $0.7998 \pm 0.0002$ & 1.2 & 1.1 \\
\hline $\mathrm{G}_{\text {sus }}$ & 1.15 & $1.1503 \pm 0.0003$ & 0.8 & 1.0 \\
\hline$\omega_{1}^{2}$ & $-1.1 \times 10^{-6}$ & $(-1.25 \pm 0.09) \times 10^{-6}$ & 1.7 & 1.0 \\
\hline$\omega_{2}^{2}$ & $-2.2 \times 10^{-6}$ & $(-2.35 \pm 0.09) \times 10^{-6}$ & 1.7 & 1.0 \\
\hline$\delta_{21}$ & $1.35 \times 10^{-4}$ & $(1.350 \pm 0.001) \times 10^{-4}$ & 0.3 & 1.0 \\
\hline$\Delta \omega^{2}$ & $-1.1 \times 10^{-6}$ & $(-1.0999 \pm 0.0003) \times 10^{-6}$ & 0.2 & 1.0 \\
\hline
\end{tabular}

implementation 23 ,

$$
T=\left\{\begin{array}{cc}
10^{\xi\left(1-\frac{\mathrm{T}_{\mathrm{h}}}{\mathrm{T}_{\mathrm{c}}}\right)} & 1 \leq i \leq \mathrm{T}_{\mathrm{h}} \\
10^{\xi\left(1-\frac{i}{\mathrm{~T}_{\mathrm{c}}}\right)} & \mathrm{T}_{\mathrm{h}} \leq i \leq \mathrm{T}_{\mathrm{c}} \\
1 & i \geq \mathrm{T}_{\mathrm{c}}
\end{array}\right.
$$

with $i$ indexing the samples of the Metropolis chain. We initially applied a constant temperature (with $\xi=3$ ) for the first 1000 iterations $\left(\mathrm{T}_{\mathrm{h}}=1000\right)$, which was then exponentially annealed down in the following 1000 iterations $\left(T_{c}=2000\right)$, after which the algorithm was properly generating samples from the actual posterior distribution. To reduce the time required during the search phase we occasionally rescale the covariance matrix of the proposal distribution to explore a wider region of the parameter space. Also, as proposed in [15, 23], we correct the standard deviation of the proposal distribution with a factor of $\mathrm{d}^{-1 / 2}$, where $\mathrm{d}$ is the parameter space dimension.

\section{E. Results and discussion}

Figure 4 illustrates the marginal posterior probability density functions of the individual parameters based on the different experiments. Parameter estimates are shown in Table IV] together with a comparison of the estimated error and the Cramér-Rao bounds, as derived in Section IIIB. The parameters are recovered successfully with estimation uncertainties roughly following the corresponding CRB, as shown in the last column of tableIV. The worse estimate appears to be $\mathrm{a} \sim 2 \sigma$ deviation on the $G_{\mathrm{df}}$ parameter in experiment 2. This result is still consistent with the true value used to generate the data. However, to further investigate this feature we generated a new set of data using the same tools and parameters. The analysis of the new data did not reproduce an offset estimate, whence we discarded a systematic bias on $G_{\mathrm{df}}$ parameter in experiment 2 .

As expected, the best estimates come from the first experiment since the signal is richer in that case. The fact that a signal is injected on both channels makes this experiment the most sensitive in terms of the determination of the stiffness difference between both test masses, reaching indeed the $\mathrm{CRB}$, and obviously translating into a better estimate for the remaining parameters.

Only the second experiment allows a better estimation of one of the parameters, $\delta_{21}$, since in this case we are canceling the second cross-coupling term, $\omega_{2}^{2}-\omega_{1}^{2}$, by forcing stiffnesses from both test masses to have the same value. As expected, the absolute value of the stiffness can not be determined accurately in such a case. The reason being that the matched stiffness configuration is precisely designed to make the experiment insensitive to stiffness differences, which naturally turns into a poor estimation of the parameter. It is however remarkable that, thanks to the cross-variance terms, we can have a good determination of the difference between the two 
stiffnesses, which should be identically zero in this case. That's indeed the value retrieved by our analysis with an uncertainty of $7 \times 10^{-10} \mathrm{~s}^{-2}$.

It is worth comparing here the results obtained with the analysis to measured quantities. Although the numerical values may differ, it may be relevant to compare the uncertainties of the values in order to check that our model is in quantitative agreement with experiments being performed. To do so we take the stiffness as our figure of merit since it has been extensively characterized in the torsion pendulum facility [24]. Recent experiments in this facility report a remnant stiffness coupling the test masses to the surrounding GRS prototype of $(2.5 \pm 0.1) \times 10^{9} \mathrm{~N} / \mathrm{m}$ [25]. When scaled by the mass of the LTP test masses $(1.96 \mathrm{~kg})$ so to be expressed in terms of force per unit mass, these figure becomes $(1.28 \pm 0.05) \times 10^{9} \mathrm{~s}^{-2}$, which could be compared to the uncertainty in the estimation of the stiffness in our model, which reaches $3 \times 10^{-10} \mathrm{~s}^{-2}$ for the second test mass stiffness in experiment 1 . The simplified noise model that we used for the analysis therefore seems to be consistent with the numbers coming from experiments. Both numbers are, however, orders of magnitude below the required remnant stiffness on board the satellite of $14 \times 10^{-7} \mathrm{~s}^{-2}[26$ ].

The data analysis during LISA Pathfinder operations will be strongly conditioned by the operations schedule. In Section III C we describe how to exploit the posterior distribution in order to combine results from different experiments. We applied that scheme to our results in order to produce a unique set of parameters for both cases previously described: all parameters being identical (experiment $1 \& 3$ ) and experiments with different numerical values of the parameters (combining all experiments). Given the approximately normal distribution of the parameters that we get from the Monte Carlo integration in Figure 4, it is justified to apply the Gaussian formalism that we introduced in Section III C. In particular, we just need to apply Equation 25 to our set of experiments. Results in Table [V] show an improvement in the uncertainty of the estimate. According to (21, the same scheme could be obtained by considering the posterior distribution of one experiment as a prior for the following one. This would also improve the convergence time of the search, which could be an important consideration during operations.

\section{SUMMARY AND FUTURE WORK}

We have shown how a Markov chain Monte Carlo method can be used for parameter estimation in the LISA Pathfinder mission. In order to demonstrate so, we generated data from a simplified model of the main experiment on board the mission, the LTP. This data set contains runs where we injected signals to test the instrument, which must allow the recovery of the parameters, and also some runs without any injection, used to evaluate
TABLE V. Combination of results for different experiments. Two values are reported when combining all experiments for parameters $\omega_{1}^{2}, \omega_{1}^{2}$ and $\Delta \omega^{2}$. The top one is the result obtained by combining the values for experiment $1 \& 3$, the bottom one corresponds to the matched stiffness experiment.

\begin{tabular}{lc|c}
\hline \hline Parameter & \multicolumn{2}{c}{ Estimated } \\
\hline $\mathrm{G}_{\mathrm{df}}$ & $0.80002 \pm 0.00002$ & $0.80003 \pm 0.00001$ \\
$\mathrm{G}_{\mathrm{sus}}$ & $1.1500001 \pm 0.0000003$ & $1.1500009 \pm 0.0000003$ \\
$\omega_{1}^{2}$ & $(-1.1000 \pm 0.0004)$ & $(-1.1000 \pm 0.0004)$ \\
$\left(\times 10^{-6}\right)$ & & $(-5 \pm 3)$ \\
$\omega_{2}^{2}$ & $(-2.2001 \pm 0.0003)$ & $(-2.2000 \pm 0.0003)$ \\
$\left(\times 10^{-6}\right)$ & & $(-5 \pm 3)$ \\
$\delta_{12}$ & $(1.3498 \pm 0.0005)$ & $(1.34967 \pm 0.00002)$ \\
$\left(\times 10^{-4}\right)$ & & \\
$\Delta \omega^{2}$ & $(-1.1002 \pm 0.0002)$ & $(-1.1002 \pm 0.0002)$ \\
$\left(\times 10^{-6}\right)$ & & $(-0.0003 \pm 0.0006)$ \\
\hline \hline
\end{tabular}

the noise performance of the instrument. We think that the model used in our analysis serves as a complementary approach to the already existing LISA simulators, since it includes some more detail in the test mass dynamics and its coupling to the test mass motion, precisely one of the key points that LISA Pathfinder aims to investigate.

The analysis presented here includes an estimate of the optimal error achievable (for an unbiased estimate) for a given injected signal and a configuration of the experiment. These results are of relevance for the mission since they show that it is as important to develop data analysis tools as to to carefully design the experiment to be performed in flight. With our model, a different injection signal showed to improve two orders of magnitude the estimation of the test mass stiffnesses - results for experiment 1 and 3 in Table II] Although the expected parameter uncertainties in the real mission will be larger than the ones reported here, the dependencies on the parameters are representative. Thus, the decrease on the optimal error could be applicable to the real mission as well. We will need however to confirm this result with more realistic models.

The method developed here to analyse the data reaches roughly the optimal attainable error for each single experiment. The combination of the results for different experiments obviously reduces the uncertainty on the parameters, reaching lower errors than the ones originally derived from the Cramér-Rao bound for each independent experiment. When combining different experiments, our analysis took advantage of the gaussian posterior ob- 
tained during the sampling of the likelihood surface, so that a simple algebraic operation between gaussian distribution was enough to derive a combined estimate of all experiments. However, the framework is general enough to include non-gaussian profiles, given that the full profile of the posterior is obtained during the sampling of the likelihood surface.

The combination of estimates was performed here as an off-line operation, i.e. after all experiments were analysed. A natural extension to this work would be to use the posterior distribution for a given experiment as prior for the next one, as motivated in Equation (21). This concept of a chain of experiments is particularly suitable for LISA Pathfinder since, during flight operations, we will naturally need to include results of previous experiments in the next foreseen ones. In other words, if the test mass stiffnesses are clearly determined in an experiment we may want to use that information for forthcoming experiments in order to effectively reduce the dimension of our problem. The method described here provides a way to include this information in the analysis in a clear way. Moreover, the capability to use this information could be a powerful advantage during operations due to the reduction of convergence time that it implies.

An increase in the uncertainty on the estimates is to be expected when dealing with a more realistic model due to the increase in dimensions of the parameter space. This is precisely the step that we will face in the forthcoming activities in preparation for the LTP data analysis. Our aim is to study in detail the experiments defined to be implemented in flight, now that the basic functionality of the parameter estimation tool is already demonstrated. In that sense, next steps will include a three dimensions model and more complex injected signals, that will make use of the full capabilities of the spacecraft. This work is ongoing and will be presented in due time.

\section{ACKNOWLEDGMENTS}

We would like to thank Curt Cutler and Ed Porter for very fruitful discussions in differents stages of this work. MN wants to thank a grant from Generalitat de Catalunya.
[1] M. Armano et al., Class. Quant. Grav. 26, 094001 (2009).

[2] P. Bender et al., Laser Interferometer Space Antenna: a cornerstone mission for the observation of gravitational waves, Tech. Rep. ESA-SCI(2000)11 (ESA, 2000).

[3] R. Dolesi, D. Bortoluzzi, P. Bosetti, L. Carbone, A. Cavalleri, I. Cristofolini, M. DaLio, G. Fontana, V. Fontanari, B. Foulon, C. D. Hoyle, M. Hueller, F. Nappo, P. Sarra, D. N. A. Shaul, T. Sumner, W. J. Weber, and S. Vitale, Classical and Quantum Gravity 20, S99 (2003), http://stacks.iop.org/0264-9381/20/i=10/a=312

[4] G. Heinzel, C. Braxmaier, M. Caldwell, K. Danzmann, F. Draaisma, A. Garcia, J. Hough, O. Jennrich, U. Johann, C. Killow, K. Middleton, M. te Plate, D. Robertson, A. Rudiger, R. Schilling, F. Steier, V. Wand, and H. Ward, Classical and Quantum Gravity 22, S149 (2005), http://stacks.iop.org/0264-9381/22/S149.

[5] M. Hewitson et al., Class. Quant. Grav. 26, 094003 (2009).

[6] A. Monsky et al., Class. Quant. Grav. 26, 094004 (2009).

[7] L. J. Rubbo, N. J. Cornish, and O. Poujade, Phys.Rev. D69, 082003 (2004), gr-qc/0311069, http://arxiv.org/ abs/gr-qc/0311069

[8] M. Vallisneri, Phys.Rev. D71, 022001 (2005), grqc/0407102, http://arxiv.org/abs/gr-qc/0407102.

[9] A. Petiteau, G. Auger, H. Halloin, O. Jeannin, E. Plagnol, S. Pireaux, T. Regimbau, and J.-Y. Vinet, Phys.Rev.D 77, 023002 (2008), 0802.2023, http:// arxiv.org/abs/0802.2023.

[10] The MathWorks, http://www . mathworks.com/.

[11] W. Fichter, P. Gath, S. Vitale, and D. Bortoluzzi, Classical and Quantum Gravity 22, S139 (2005), http:// stacks. iop.org/0264-9381/22/i=10/a=002.

[12] D. Bortoluzzi, L. Carbone, A. Cavalleri, M. D. Lio, R. Dolesi, C. D. Hoyle, M. Hueller, S. Vitale, and W. J.
Weber, Classical and Quantum Gravity 21, S573 (2004), http://stacks.iop.org/0264-9381/21/i=5/a=028.

[13] S. Vitale, Measurement of LTP dynamical coefficients by system identification, Tech. Rep. S2-UTN-TN-3045 Issue 2 (2008).

[14] L. Ferraioli, G. Heinzel, M. Hewitson, M. Hueller, A. Monsky, M. Nofrarias, and S. Vitale 1006.0138, http: //arxiv.org/abs/1006.0138

[15] A. Gelman, J. B. Carlin, H. Stern, and D. B. Rubin, Bayesian data analysis (Chapman \& Hall / CRC, 1997).

[16] P. C. Gregory, Bayesian logical data analysis for the physical sciences (Cambridge University Press, 2005).

[17] M. Vallisneri, Phys.Rev.D 77, 042001 (2008), grqc/0703086, http://arxiv.org/abs/gr-qc/0703086

[18] A. Zeira and A. Nehorai, IEEE Trans. Acoust., Speech and Sig. Proc. 38, 1063 (1990).

[19] P. Welch, IEEE Trans. Audio and Electroacoust. 15, 70 (1967).

[20] D. M. Himmelblau, Applied nonlinear programming (McGraw-Hill, New York, 1972).

[21] W. R. Gilks, S. Richardson, and D. J. Spiegelhalter, Markov chain Monte Carlo in practice (Chapman \& Hall / CRC, 1996).

[22] C. Röver, Bayesian inference on astrophysical binary inspirals based on gravitational-wave measurements, Ph.D. thesis, The University of Auckland (2007), URL: http: //hdl.handle.net/2292/2356

[23] N. J. Cornish and E. K. Porter, Phys.Rev.D 75, 021301 (2007), gr-qc/0605135, http://arxiv.org/abs/gr-qc/ 0605135

[24] L. Carbone, A. Cavalleri, R. Dolesi, C. D. Hoyle, M. Hueller, S. Vitale, and W. J. Weber, Phys. Rev. Lett. 91, 151101 (Oct 2003).

[25] A. Cavalleri, G. Ciani, R. Dolesi, M. Hueller, D. Nicolodi, 
D. Tombolato, P. J. Wass, W. J. Weber, S. Vitale, and L. Carbone, Classical and Quantum Gravity 26, 094012 (10pp) (2009), http://stacks .iop.org/0264-9381/26/ 094012 .
[26] S. Vitale, Science requirements and top-level architecture definition for the LTP on board LISA Pathfinder, Tech. Rep. LTPA-UTN-ScRD Issue 3 (2005). 
Experiment 1
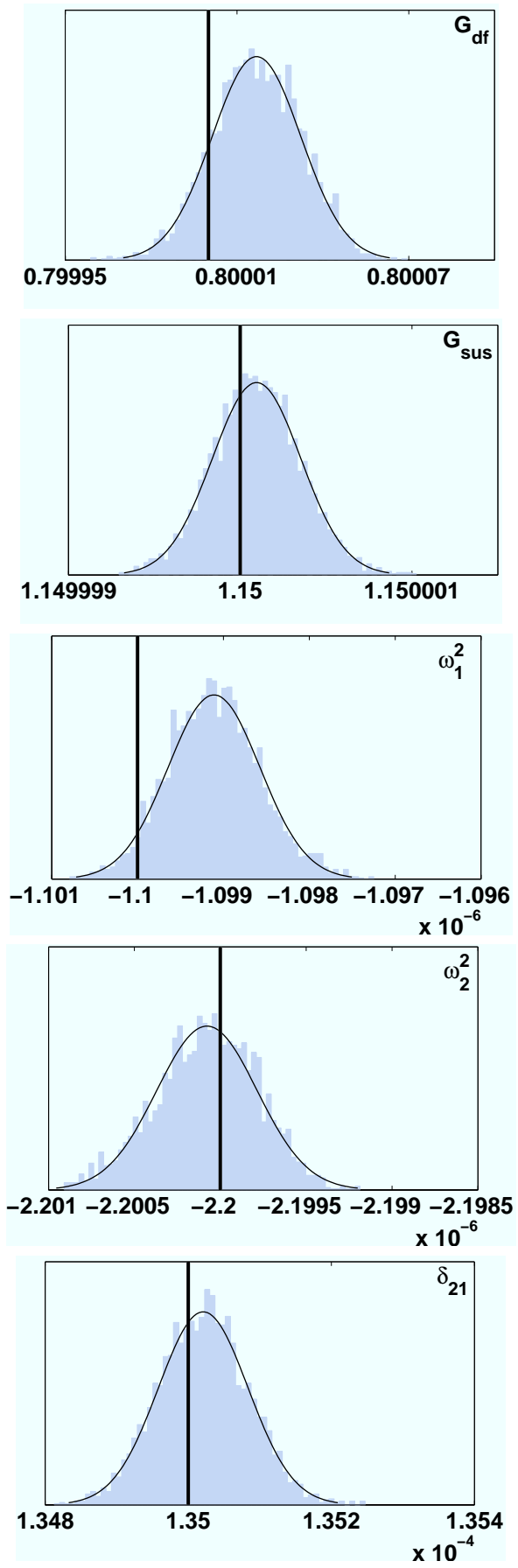

Experiment 2
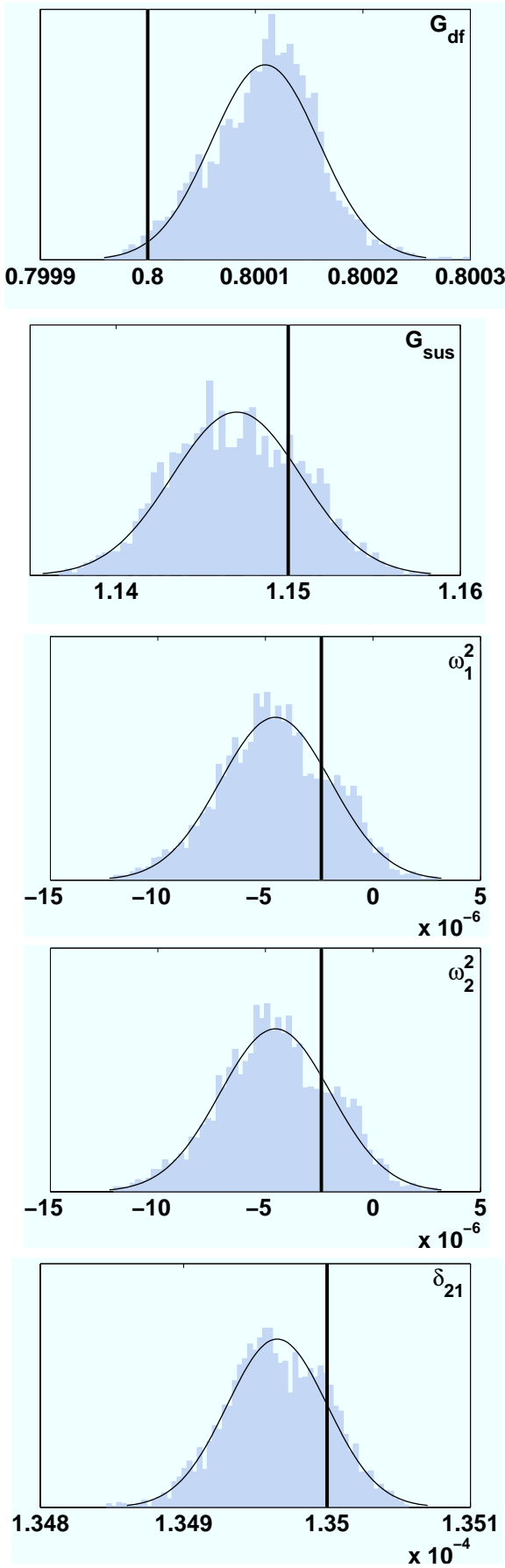

Experiment 3
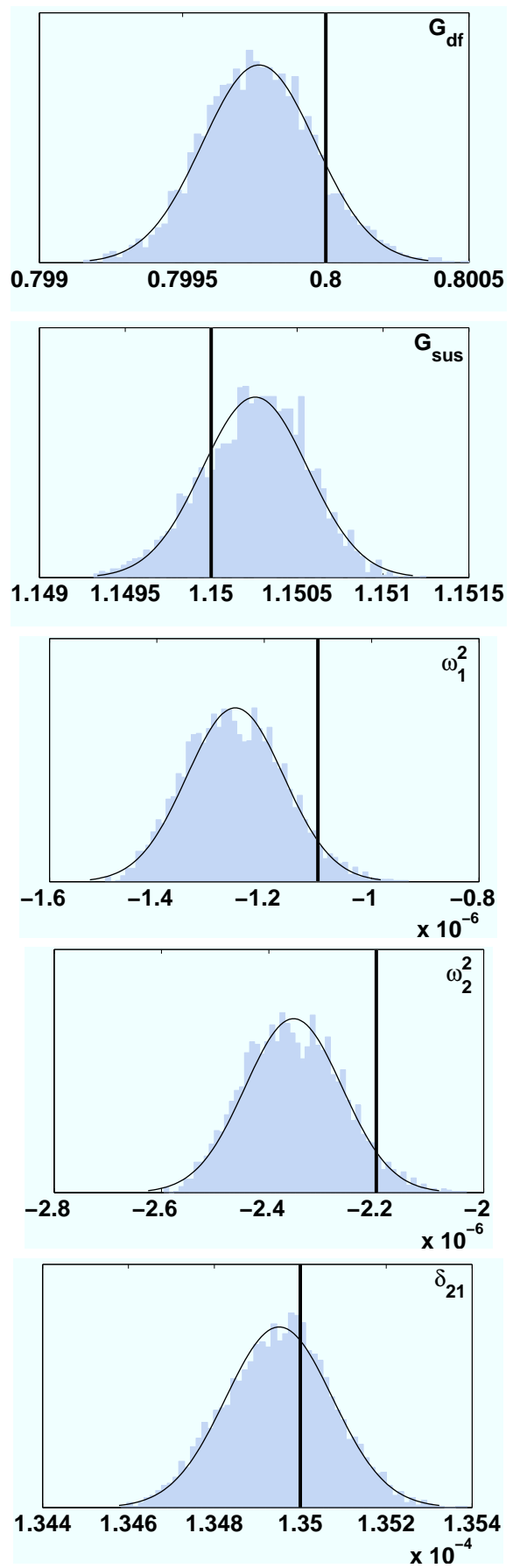

FIG. 4. Histograms of the MCMC samples illustrating the individual parameters' marginal posterior probability distributions as computed with the last 3500 samples of the chain. All histograms are plot with the same y axes range, up to 250 counts. Black vertical lines illustrate the true parameter values. Parameters $G_{\mathrm{df}}, G_{\mathrm{sus}}$ and $\delta_{21}$ are dimensionless; dimensions for stiffness parameters are $\left[\omega_{1}^{2}\right]=\left[\omega_{2}^{2}\right]=\mathrm{s}^{-2}$. 